\title{
ON DIFFERENTIAL GEOMETRY OF HYPERSURFACES IN THE LARGE
}

\author{
BY \\ CHUAN-CHIH HSIUNG
}

1. Introduction. Let $V^{n}\left(V^{*_{n}}\right)$ be an orientable hypersurface of class $C^{3}$ imbedded in a Euclidean space $E^{n+1}$ of $n+1 \geqq 3$ dimensions with a closed boundary $V^{n-1}\left(V^{*-1}\right)$ of dimension $n-1$. Suppose that there is a one-to-one correspondence between the points of the two hypersurfaces $V^{n}, V^{*_{n}}$ such that at corresponding points the two hypersurfaces $V^{n}, V^{* n}$ have the same normal vectors. Let $\kappa_{1}, \cdots, \kappa_{n}$ be the $n$ principal curvatures at a point $P$ of the hypersurface $V^{n}$, then the $\alpha$ th mean curvature $M_{\alpha}$ of the hypersurface $V^{n}$ at the point $P$ is defined by

$$
\left(\begin{array}{l}
n \\
\alpha
\end{array}\right) M_{\alpha}=\sum \kappa_{1} \cdots \kappa_{\alpha} \quad(\alpha=1, \cdots, n),
$$

where the expression on the right side is the $\alpha$ th elementary symmetric function of $\kappa_{1}, \cdots, \kappa_{n}$. In particular, $M_{n}$ is the Gaussian curvature of the hypersurface $V^{n}$ at the point $P$. Let $P^{*}$ be the point of the hypersurface $V^{*_{n}}$ corresponding to the point $P$ of the hypersurface $V^{n}$ under the given correspondence, $p^{*}$ the oriented distance from a fixed point $O$ in the space $E^{n+1}$ to the tangent hyperplane of the hypersurface $V^{*_{n}}$ at the point $P^{*}$, and $d A$ the area element of the hypersurface $V^{n}$ at the point $P$. The purpose of this paper is first to derive some expressions for the integrals $\int_{V^{n}} M_{\alpha} p^{*} d A(\alpha=1, \cdots, n)$, and then to prove the following

Theorem. Let $V^{n}\left(V^{* n}\right)$ be an orientable hypersurface of class $C^{3}$ imbedded in a Euclidean space $E^{n+1}$ of $n+1 \geqq 3$ dimensions with a positive Gaussian curvature and a closed boundary $V^{n-1}\left(V^{*_{n-1}}\right)$ of dimension $n-1$. Suppose that there is a one-to-one correspondence between the points of the two hypersurfaces $V^{n}, V^{*_{n}}$, such that at corresponding points the two hypersurfaces $V^{n}, V^{*_{n}}$ have the same normal vectors and equal sums of the principal radii of curvature, and such that the two boundaries $V^{n-1}, V^{* n-1}$ are congruent. Then the two hypersurfaces $V^{n}, V^{* n}$ are congruent or symmetric.

This theorem has been obtained by T. Kubota (see [6] or [1, pp. 29-30]) for closed hypersurfaces $V^{n}, V^{* n}$, and by the author [5] for $n=2$ in a slightly different form.

2. Preliminaries. In a Euclidean space $E^{n+1}$ of dimension $n+1 \geqq 3$, let us

Received by the editors May 8, 1955. 
consider a fixed orthogonal frame $O \mathfrak{y}_{1} \cdots \mathfrak{Y}_{n+1}$ with a point $O$ as the origin. With respect to this orthogonal frame we define the vector product of $n$ vectors $A_{1}, \cdots, A_{n}$ in $E^{n+1}$ to be the vector $A_{n+1}$, denoted by $A_{1} \times \cdots \times A_{n}$, satisfying the following conditions:

(a) the vector $A_{n+1}$ is normal to the $n$-dimensional space determined by the vectors $A_{1}, \cdots, A_{n}$,

(b) the magnitude of the vector $A_{n+1}$ is equal to the volume of the parallelepiped whose edges are the vectors $A_{1}, \cdots, A_{n}$,

(c) the two frames $O A_{1} \cdots A_{n} A_{n+1}$ and $O \mathfrak{Y}_{1} \cdots \mathfrak{Y}_{n+1}$ have the same orientation.

Let $\sigma$ be a permutation on the $n$ numbers $1, \cdots, n$, then

$$
A_{\sigma(1)} \times \cdots \times A_{\sigma(n)}=(\operatorname{sgn} \sigma) A_{1} \times \cdots \times A_{n},
$$

where $\operatorname{sgn} \sigma$ is +1 or -1 according as the permutation $\sigma$ is even or odd. Let $i_{1}, \cdots, i_{n+1}$ be the unit vectors from the origin $O$ in the directions of the vectors $\mathfrak{Y}_{1}, \cdots, \mathfrak{Y}_{n+1}$ and let $A_{\alpha}^{j}(j=1, \cdots, n+1)$ be the components of the vector $A_{\alpha}(\alpha=1, \cdots, n)\left({ }^{1}\right)$ with respect to the frame $O \mathfrak{Y}_{1} \cdots \mathfrak{Y}_{n+1}$, then the scalar product of any two vectors $A_{\alpha}$ and $A_{\beta}$ and the vector product of $n$ vectors $A_{1}, \cdots, A_{n}$ are, respectively,

$$
\begin{aligned}
& A_{\alpha} \cdot A_{\beta}=\sum_{i=1}^{n+1} A_{\alpha}^{i} A_{\beta}^{i},
\end{aligned}
$$

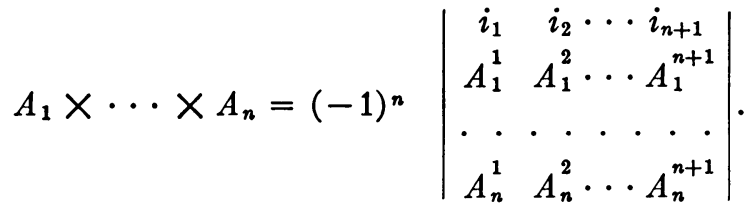

If $A_{\alpha}^{j}$ are differentiable functions of $n$ variables $x^{1}, \cdots, x^{n}$, then by equation (2.3) and the differentiation of determinants

(2.4) $\frac{\partial}{\partial x^{\alpha}}\left(A_{1} \times \cdots \times A_{n}\right)=\sum_{\beta=1}^{n}\left(A_{1} \times \cdots \times A_{\beta-1} \times \frac{\partial A_{\beta}}{\partial x^{\alpha}} \times A_{\beta+1} \times \cdots \times A_{n}\right)$.

Now we consider a hypersurface $V^{n}$ of class $C^{3}$ imbedded in the space $E^{n+1}$ with a closed boundary $V^{n-1}$ of dimension $n-1$. Let $\left(y^{1}, \cdots, y^{n+1}\right)$ be the coordinates of a point $P$ in the space $E^{n+1}$ with respect to the orthogonal frame $O \mathfrak{Y}_{1} \cdots \mathfrak{Y}_{n+1}$. Then the hypersurface $V^{n}$ can be given by the parametric equations $\left({ }^{2}\right)$

(1) Throughout this paper all Latin indices take the values 1 to $n+1$ and Greek indices the values 1 to $n$ unless stated otherwise. We shall also follow the convention that repeated indices imply summation.

(2) For the remainder of this section see, for instance, [2, Chap. IV]. 


$$
y^{i}=f^{i}\left(x^{1}, \cdots, x^{n}\right) \quad(i=1, \cdots, n+1),
$$

or the vector equation

$$
Y=F\left(x^{1}, \cdots, x^{n}\right),
$$

where $y^{i}$ and $f^{i}$ are respectively the components of the two vectors $Y$ and $F$, the parameters $x^{1}, \cdots, x^{n}$ take values in a simply connected domain $D$ of the $n$-dimensional real number space, $f^{i}\left(x^{1}, \cdots, x^{n}\right)$ are of the third class and the Jacobian matrix $\left\|\partial y^{i} / \partial x^{\alpha}\right\|$ is of rank $n$ at all points of $D$. If we denote the vector $\partial Y / \partial x^{\alpha}$ by $Y_{\alpha}$ for $\alpha=1, \cdots, n$, then the first fundamental form of the hypersurface $V^{n}$ at a point $P$ is

$$
d s^{2}=g_{\alpha \beta} d x^{\alpha} d x^{\beta},
$$

where

$$
g_{\alpha \beta}=Y_{\alpha} \cdot Y_{\beta},
$$

and the matrix $\left\|g_{\alpha \beta}\right\|$ is positive definite so that the determinant $g=\left|g_{\alpha \beta}\right|>0$.

Let $N$ be the unit normal vector of the hypersurface $V^{n}$ at a point $P$, and $N_{\alpha}$ the vector $\partial N / \partial x^{\alpha}$, then

$$
N_{\alpha}=-b_{\alpha \beta} g^{\beta \gamma} Y_{\gamma},
$$

where

$$
b_{\alpha \beta}=b_{\beta \alpha}=-Y_{\alpha} \cdot N_{\beta}
$$

are the coefficients of the second fundamental form of the hypersurface $V^{n}$ at the point $P$, and $g^{\beta \gamma}$ denotes the cofactor of $g_{\beta \gamma}$ in $g$ divided by $g$ so that

$$
g^{\alpha \beta} g_{\beta \gamma}=\delta_{\gamma}^{\alpha},
$$

$\delta_{\gamma}^{\alpha}$ being the Kronecker deltas. The $n$ principal curvatures $\kappa_{1}, \cdots, \kappa_{n}$ of the hypersurface $V^{n}$ at the point $P$ are the roots of the determinant equation

$$
\left|b_{\alpha \beta}-\kappa g_{\alpha \beta}\right|=0 \text {. }
$$

From equations (1.1) and (2.12) follow immediately

$$
M_{n}=b / g, \quad n M_{1}=b_{\alpha \beta} g^{\alpha \beta}, \quad n M_{n-1}=g_{\alpha \beta} B^{\alpha \beta} / g,
$$

where $b=\left|b_{\alpha \beta}\right| \neq 0$ and $B^{\alpha \beta}$ is the cofactor of $b_{\alpha \beta}$ in $b$.

The third fundamental form of the hypersurface $V^{n}$ at the point $P$ is

$$
d N \cdot d N=f_{\alpha \beta} d x^{\alpha} d x^{\beta},
$$

where we have placed

$$
f_{\alpha \beta}=N_{\alpha} \cdot N_{\beta}
$$


From equations (2.8), (2.9), and (2.11), it follows immediately that

$$
f_{\alpha \beta}=b_{\alpha \beta} b_{\beta \sigma} g^{\rho \sigma} \text {, }
$$

and therefore that

$$
g^{\alpha \beta}=f_{\rho \sigma} b^{\alpha \rho} b^{\beta \sigma},
$$

where $b^{\alpha \rho}=B^{\alpha \rho} / b$. It is easily seen that the principal radii of curvature $r_{\alpha}(\alpha=1, \cdots, n)$ of the hypersurface $V^{n}$ at the point $P$ are the roots of the determinant equation

$$
\left|b_{\alpha \beta}-r f_{\alpha \beta}\right|=0
$$

from which we obtain

$$
r_{1} \cdots r_{n}=b / f, \quad \sum_{\alpha=1}^{n} r_{\alpha}=b_{\alpha \beta} f^{\alpha \beta}, \quad \sum r_{1} \cdots r_{n-1}=f_{\alpha \beta} B^{\alpha \beta} / f,
$$

where $f^{\alpha \beta}$ denotes the cofactor of $f_{\alpha \beta}$ in $f=\left|f_{\alpha \beta}\right|$ divided by $f$. From equations (2.13) and (2.19) it follows immediately that

$$
f=M_{n}^{2} g>0 .
$$

The area element of the hypersurface $V^{n}$ at the point $P$ is given by

$$
d A=g^{1 / 2} d x^{1} \cdots d x^{n} .
$$

Now we choose the direction of the unit normal vector $N$ in such a way that the two frames $P Y_{1} \cdots Y_{n} N$ and $O \mathfrak{D}_{1} \cdots \mathfrak{Y}_{n+1}$ have the same orientation. Then from equations (2.3) and (2.21) it follows that

$$
\begin{aligned}
& g^{1 / 2} N=Y_{1} \times \cdots \times Y_{n}, \\
& \left|Y_{1}, \cdots, Y_{n}, N\right|=g^{1 / 2} .
\end{aligned}
$$

Let $u^{1}, \cdots, u^{n-1}$ be the local coordinates of a point $P$ on the boundary $V^{n-1}$, then the first fundamental form of the boundary $V^{n-1}$ at the point $P$ is

$$
d s^{2}=a_{\lambda \mu} d u^{\lambda} d u^{\mu} \quad\left(\lambda, \mu={ }_{1}, \cdots, n-1\right),
$$

where

$$
a_{\lambda \mu}=g_{\alpha \beta} \frac{\partial x^{\alpha}}{\partial u^{\lambda}} \frac{\partial x^{\beta}}{\partial u^{\mu}}
$$

and the matrix $\left\|a_{\lambda_{\mu}}\right\|$ is positive definite so that the determinant $a=\left|a_{\lambda \mu}\right|>0$. The coefficients of the second fundamental form of the boundary $V^{n-1}$ corresponding to the unit normal vector $N$ of the hypersurface $V^{n}$ at the point $P$ are 


$$
\Omega_{\lambda \mu}=\sum_{i=1}^{n+1} N^{i}\left(\frac{\partial^{2} y^{i}}{\partial u^{\lambda} \partial u^{\mu}}-\left\{\begin{array}{c}
\nu \\
\lambda \mu
\end{array}\right\} \cdot \frac{\partial y^{i}}{\partial u^{\nu}}\right) \quad(\lambda, \mu, \nu=1, \cdots, n-1),
$$

where

$$
\left\{\begin{array}{c}
\nu \\
\lambda \mu
\end{array}\right\}_{a}
$$

is a Christoffel symbol of the second kind formed with respect to the $a$ 's and $u$ 's. Similarly, for the hypersurface $V^{n}$ we have

$$
b_{\alpha \beta} N=\frac{\partial^{2} Y}{\partial x^{\alpha} \partial x^{\beta}}-\left\{\begin{array}{c}
\gamma \\
\alpha \beta
\end{array}\right\}_{0} Y_{\gamma},
$$

where

$$
\left\{\begin{array}{c}
\gamma \\
\alpha \beta
\end{array}\right\}
$$

is a Christoffel symbol of the second kind formed with respect to the $g$ 's and $x$ 's. From equations (2.26) and (2.27) it is easily seen that

$$
\Omega_{\lambda \mu}=b_{\alpha \beta} \frac{\partial x^{\alpha}}{\partial u^{\lambda}} \frac{\partial x^{\beta}}{\partial u^{\mu}} .
$$

3. Some integral formulas. Suppose that there is a one-to-one correspondence between the points of two hypersurfaces $V^{n}, V^{*_{n}}$ of class $C^{3}$ imbedded in a space $E^{n+1}$ with positive Gaussian curvatures and closed boundaries $V^{n-1}, V^{* n-1}$ of dimension $n-1$ respectively such that the two hypersurfaces $V^{n}, V^{*_{n}}$ have the same normal vectors at corresponding points. Without loss of generality we may assume that the corresponding points of the two hypersurfaces $V^{n}, V^{*_{n}}$ have the same local coordinates $x^{1}, \cdots, x^{n}$. Then $\$ 2$ can be applied to the hypersurface $V^{n}$, and for the corresponding quantities for the hypersurface $V^{*_{n}}$ we shall use the same symbols with a star.

At first, we observe that the vector $Y_{1} \times \cdots \times Y_{\alpha-1} \times N \times Y_{\alpha+1} \times \cdots$ $\times Y_{n}$ is perpendicular to the normal vector $N$ and that the $n$ vectors $Y_{1}, \cdots, Y_{n}$ are linearly independent in the tangent hyperplane of the hypersurface $V^{n}$ at the point $P$. Therefore the vector $Y_{1} \times \cdots \times Y_{\alpha-1} \times N \times Y_{\alpha+1}$ $\times \cdots \times Y_{n}$ can be written in the form

$$
Y_{1} \times \cdots \times Y_{\alpha-1} \times N \times Y_{\alpha+1} \times \cdots \times Y_{n}=a^{\alpha \beta} N_{\beta} .
$$

Taking the scalar products of the both sides of equations (3.1) with the vector $Y_{\gamma}$ and making use of equations (2.2), (2.3), (2.10), (2.23), we obtain

$$
a^{\alpha \beta} b_{\beta \gamma}=g^{1 / 2} \delta_{\gamma}^{\alpha} \quad(\alpha, \gamma=1, \cdots, n) .
$$

Solving equations (3.2) for $a^{\alpha \beta}$ for each fixed $\alpha$ and substituting the results in 
equations (3.1), we are led to

$$
Y_{1} \times \cdots \times Y_{\alpha-1} \times N \times Y_{\alpha+1} \times \cdots \times Y_{n}=g^{1 / 2} b^{\alpha \beta} N_{\beta} .
$$

Making use of equations (2.4), (2.9), (2.13), (2.22) and the relation

$$
\begin{array}{r}
Y_{1} \times \cdots \times Y_{\beta-1} \times Y_{\beta \alpha} \times Y_{\beta+1} \times \cdots \times Y_{\alpha-1} \times N \times Y_{\alpha+1} \times \cdots \times Y_{n} \\
=-Y_{1} \times \cdots \times Y_{\beta-1} \times N \times Y_{\beta+1} \times \cdots \times Y_{\alpha-1} \times Y_{\alpha \beta} \times Y_{\alpha+1} \times \cdots \times Y_{n} \\
(\alpha>\beta ; \alpha, \beta=1, \cdots, n),
\end{array}
$$

it is easily seen that

$$
\begin{aligned}
& \sum_{\alpha=1}^{n} \frac{\partial}{\partial x^{\alpha}}\left(Y_{1} \times \cdots \times Y_{\alpha-1} \times N \times Y_{\alpha+1} \times \cdots \times Y_{n}\right) \\
= & \sum_{\alpha=1}^{n} Y_{1} \times \cdots \times Y_{\alpha-1} \times N_{\alpha} \times Y_{\alpha+1} \times \cdots \times Y_{n}=-n g^{1 / 2} M_{1} N .
\end{aligned}
$$

Thus, from equations (3.3) and (3.4),

$$
n g^{1 / 2} M_{1} N=-\frac{\partial}{\partial x^{\alpha}}\left(g^{1 / 2} b^{\alpha \beta} N_{\beta}\right) .
$$

Taking the scalar products of the both sides of equation (3.5) with the position vector $Y^{*}$ of the corresponding point $P^{*}$ of the point $P$, we obtain in consequence of the relation $b^{2}=f g$, obtained from equations (2.13) and (2.19),

$$
n M_{1} g^{1 / 2} p^{*}=\frac{1}{f^{1 / 2}} \sum_{\alpha=1}^{n} B^{\alpha \beta} N_{\beta} \cdot Y_{\alpha}^{*}-\sum_{\alpha=1}^{n} \frac{\partial}{\partial x^{\alpha}}\left(\frac{1}{f^{1 / 2}} B^{\alpha \beta} Y^{*} \cdot N_{\beta}\right),
$$

where we have placed

$$
p^{*}=Y^{*} \cdot N \text {. }
$$

Integrating equation (3.6) with respect to $x^{1}, \cdots, x^{n}$ over the hypersurface $V^{n}$ and applying the general Green's theorem (cf., for instance, [3, pp. 75-76]) to the second term on the right side of equation (3.6), we then obtain

$$
\begin{aligned}
& \int_{V^{n}} n M_{1} p^{*} d A= \int_{V^{n}} \frac{1}{f^{1 / 2}} \sum_{\alpha=1}^{n} B^{\alpha \beta} N_{\beta} \cdot Y_{\alpha}^{*} d x^{1} \cdots d x^{n} \\
&-\int_{V^{n-1}} \frac{1}{f^{1 / 2}} \sum_{\alpha=1}^{n}(-1)^{\alpha-1} B^{\alpha \beta} Y^{*} \\
& \cdot N_{\beta} d x^{1} \cdots d x^{\alpha-1} d x^{\alpha+1} \cdots d x^{n} .
\end{aligned}
$$

In order to use the formula (3.8) to derive an analogous expression for the integral $\int_{V^{n}} M_{\alpha} P^{*} d A$ for a general $\alpha(\alpha=1, \cdots, n)$, in the space $E^{n+1}$ we first consider a hypersurface $\bar{V}^{n}$ parallel to the hypersurface $V^{n}$ so that the two hypersurfaces $V^{n}$; $\bar{V}^{n}$ have the same normals. It is evident that the 
vector equation of the hypersurface $\bar{V}^{n}$ can be written in the form

$$
\bar{Y}=Y-t N,
$$

where $t$ is a real parameter. From equations (3.9), $N \cdot N=1$ and $N \cdot \bar{Y}_{\alpha}$ $=N \cdot \partial \bar{Y} / \partial x^{\alpha}=0$, it follows immediately that $\partial t / \partial x^{\alpha}=0$ and therefore that $t$ is constant. Making use of equations (2.8), (2.10), (2.15) and their analogous ones for the hypersurface $\bar{V}^{n}$, we obtain the coefficients of the first and the second fundamental forms of the hypersurface $\bar{V}^{n}$ :

$$
\begin{aligned}
& \bar{g}_{\alpha \beta}=g_{\alpha \beta}+2 b_{\alpha \beta} t+f_{\alpha \beta} t^{2}, \\
& b_{\alpha \beta}=b_{\alpha \beta}+f_{\alpha \beta} t,
\end{aligned}
$$

from which it follows easily by an elementary calculation that

$$
\begin{aligned}
b & =b \Delta, \\
\bar{g} & =g \Delta^{2}, \\
\left|\bar{r} b_{\alpha \beta}-\bar{g}_{\alpha \beta}\right| & =\left|(\bar{r}-t) b_{\alpha \beta}-g_{\alpha \beta}\right| \Delta,
\end{aligned}
$$

where $\bar{g}=\left|\bar{g}_{\alpha \beta}\right|, \bar{b}=\left|\delta_{\alpha \beta}\right|$ and

$\bar{\kappa}_{\alpha}$ being the principal curvatures of the hypersurface $\bar{V}^{n}$. In consequence of equations (3.12), (3.13), (3.14) and (2.12), (2.13), (2.21) together with their analogues for the hypersurface $\bar{V}^{n}$, we have

$$
\begin{aligned}
& \Delta=\left|\delta_{\alpha}^{\beta}+b_{\alpha \rho} g^{\rho \beta} t\right|, \\
& \bar{r}_{\alpha}=1 / \bar{\kappa}_{\alpha} \\
& \text { atures of the hypersu } \\
& 14) \text { and }(2.12),(2.13) \\
& \qquad \begin{aligned}
\bar{M}_{n} d \bar{A}=M_{n} d A, \\
\bar{\gamma}_{\alpha}=r_{\alpha}+t,
\end{aligned}
\end{aligned}
$$$$
(\alpha=1, \cdots, n),
$$

where $d \bar{A}$ is the area element of the hypersurface $\bar{V}^{n}$.

Similarly, let $\bar{V}^{*_{n}}$ be a hypersurface in the space $E^{n+1}$ parallel to the hypersurface $V^{* n}$ and having the vector equation

$$
\bar{Y}^{*}=Y^{*}-t N,
$$

where $t$ is the same arbitrary constant as in equation (3.9). For this one-to-one correspondence between the points of the two hypersurfaces $\bar{V}^{n}, \bar{V}^{* n}$, equation (3.8) can be written as, by means of equations (1.1) and (3.16),

$$
\begin{aligned}
\int_{\bar{V}^{n}} F^{*}( & \left.\sum \bar{\gamma}_{1} \bar{\gamma}_{2} \cdots \bar{r}_{n-1}\right) \bar{M}_{n} d \bar{A} \\
= & \int_{\bar{V}^{n}} \frac{1}{f^{1 / 2}} \sum_{\alpha=1}^{n} \bar{B}^{\alpha \beta} N_{\beta} \cdot \bar{Y}_{\alpha}^{*} d x^{1} \cdots d x^{n} \\
& -\int_{\bar{V}^{n-1}} \frac{1}{f^{1 / 2}} \sum_{\alpha=1}^{n}(-1)^{\alpha-1} \bar{B}^{\alpha \beta} N_{\beta} \cdot \bar{Y}^{*} d x^{1} \cdots d x^{\alpha-1} d x^{\alpha+1} \cdots d x^{n},
\end{aligned}
$$


where $\bar{p}^{*}=\bar{Y}^{*} \cdot N=p^{*}-t, \bar{B}^{\alpha \beta}$ is the cofactor of $b_{\alpha \beta}$ in $b, \bar{Y}_{\alpha}^{*}=\partial \bar{Y}^{*} / \partial x^{\alpha}$, and $\bar{V}^{n-1}$ is the boundary of the hypersurface $\bar{V}^{n}$. Substitution, in equation (3.20), of equations (3.17), (3.18), (2.15) and the analogue of equation (2.10) for the hypersurface $V^{*_{n}}$ yields immediately

$$
\begin{aligned}
\int_{V^{n}}\left(p^{*}-t\right) \sum_{\alpha=1}^{n}(n-\alpha+1)\left(\sum r_{1} \cdots r_{\alpha-1}\right) t^{n-\alpha} M_{n} d A \\
=-\int_{V^{n}} \frac{1}{f^{1 / 2}} \sum_{\alpha=1}^{n} \bar{B}^{\alpha \beta}\left(b_{\beta \alpha}^{*}+t f_{\alpha \beta}\right) d x^{1} \cdots d x^{n} \\
\quad+\int_{V^{n-1}} \frac{1}{f^{1 / 2}} \sum_{\alpha=1}^{n}(-1)^{\alpha-1} Y^{*} \cdot N_{\beta} \bar{B}^{\alpha \beta} d x^{1} \cdots d x^{\alpha-1} d x^{\alpha+1} \cdots d x^{n},
\end{aligned}
$$

which is an identity in $t$. Hence, by equating the coefficients of $t^{0}, t, \cdots, t^{n-1}$ on the both sides of equation (3.21) and using equation (1.1), we can obtain $n$ formulas, one of which is equation (3.8). These $n$ formulas have been obtained by the author [4] for the case where the two hypersurfaces $V^{n}, V^{* n}$ coincide.

4. Proof of the theorem. In order to prove the theorem stated in the introduction, we may assume, for simplicity, that the local coordinate $x^{1}, \cdots, x^{n}$ of the two hypersurfaces $V^{n}, V^{*_{n}}$ be so chosen that

$$
f_{\alpha \beta}=0 \text { for } \alpha \neq \beta \text {. }
$$

Then from equation (3.11) it follows that

$$
\bar{B}^{\alpha \beta}=\frac{f}{f_{\alpha \alpha} f_{\beta \beta}}\left[-b_{\beta \alpha} t^{n-2}+\sum_{\gamma=1, \gamma \neq \alpha, \gamma \neq \beta}^{n}\left(b_{\beta \gamma} b_{\gamma \alpha}-b_{\gamma \gamma} b_{\beta \alpha}\right) t^{n-3} / f_{\gamma \gamma}\right]+\cdots
$$
for $\beta \neq \alpha$,

$$
\begin{aligned}
\bar{B}^{\alpha \alpha}=\frac{f}{f_{\alpha \alpha}}\left[t^{n-1}+\sum_{\gamma=1, \gamma \neq \alpha}^{n}\right. & \frac{b_{\gamma \gamma}}{f_{\gamma \gamma}} t^{n-2} \\
& \left.+\frac{1}{2} \sum_{\beta, \gamma=1 ; \beta, \gamma \neq \alpha ; \beta \neq \gamma}^{n} \frac{b_{\beta \beta} b_{\gamma \gamma}-b_{\beta \gamma}^{2}}{f_{\beta \beta} f_{\gamma \gamma}} t^{n-3}\right]+\cdots,
\end{aligned}
$$

where the unwritten terms are of degrees $<n-3$ in $t$. Moreover, an elementary calculation from equation $(2.18)$ leads to

$$
\sum r_{1} r_{2}=\frac{1}{2} \sum_{\alpha, \beta=1 ; \alpha \neq \beta}^{n} \frac{b_{\alpha \alpha} b_{\beta \beta}-b_{\alpha \beta}^{2}}{f_{\alpha \alpha} f_{\beta \beta}} .
$$

Thus, by equating the coefficients of $t^{n-2}$ on the both sides of equation (3.21) and using equations (4.1), (4.2), (4.3), we obtain 


$$
\begin{aligned}
(n-1) \int_{V^{n}} p^{*}\left(\sum_{\alpha=1}^{n} r_{\alpha}\right) M_{n} d A & \\
= & -\int_{V^{n}} \sum_{\alpha, \beta=1 ; \alpha \neq \beta}^{n} \frac{f^{1 / 2}}{f_{\alpha \alpha} f_{\beta \beta}}\left(b_{\beta \beta} b_{\alpha \alpha}^{*}-b_{\alpha \beta} b_{\beta \alpha}^{*}\right) d x^{1} \cdots d x^{n} \\
& +\int_{V^{n-1}} \sum_{\alpha, \beta=1 ; \alpha \neq \beta}^{n}(-1)^{\alpha-1} \frac{f^{1 / 2}}{f_{\alpha \alpha} f_{\beta \beta}} Y^{*} \\
& \cdot\left(N_{\alpha} b_{\beta \beta}-N_{\beta} b_{\beta \alpha}\right) d x^{1} \cdots d x^{\alpha-1} d x^{\alpha+1} \cdots d x^{n} .
\end{aligned}
$$

Replacing the hypersurface $V^{n}$ by the hypersurface $V^{*_{n}}$ in equation (4.4) gives

$$
\begin{aligned}
(n-1) \int_{V^{* n}} p^{*}\left(\sum_{\alpha=1}^{n} r_{\alpha}^{*}\right) M_{n}^{*} d A^{*} & \\
= & -\int_{V^{n}} \sum_{\alpha, \beta=1 ; \alpha \neq \beta}^{n} \frac{f^{1 / 2}}{f_{\alpha \alpha} f_{\beta \beta}}\left(b_{\beta \beta}^{*} b_{\alpha \alpha}^{*}-b_{\alpha \beta}^{*} b_{\beta \alpha}^{*}\right) d x^{1} \cdots d x^{n} \\
& \quad+\int_{V^{n-1}} \sum_{\alpha, \beta-1 ; \alpha \neq \beta}^{n}(-1)^{\alpha-1} \frac{f^{1 / 2}}{f_{\alpha \alpha} f_{\beta \beta}} Y^{*} \\
& \cdot\left(N_{\alpha} b_{\beta \beta}^{*}-N_{\beta} b_{\beta \alpha}^{*}\right) d x^{1} \cdots d x^{\alpha-1} d x^{\alpha+1} \cdots d x^{n} .
\end{aligned}
$$

Since under the given one-to-one correspondence between the points of the two hypersurfaces $V^{n}, V^{* n}$, the two boundaries $V^{n-1}, V^{* n-1}$ are congruent, we may assume that the corresponding points of the boundaries $V^{n-1}$, $V^{* n-1}$ have the same local coordinates $u^{1}, \cdots, u^{n-1}$. Then the second fundamental forms of the boundaries $V^{n-1}, V^{*_{n-1}}$ corresponding to the common unit normal vector $N$ of the hypersurfaces $V^{n}, V^{*_{n}}$ at the corresponding points of the boundaries $V^{n-1}, V^{* n-1}$ are equal (see, for instance, [2, p. 192]), and therefore from equation (2.28) and its analogue for the hypersurface $V^{*_{n}}$ it follows that $b_{\alpha \beta}=b_{\alpha \beta}^{*}$ at corresponding points of the two boundaries $V^{n-1}, V^{* n-1}$. Thus the second integrals on the right side of equations (4.4), (4.5) are equal. On the other hand, by the assumption of the theorem we have $\sum_{\alpha=1}^{n} r_{\alpha}=\sum_{\alpha=1}^{n} r_{\alpha}^{*}$, and from equations (2.13), (2.19) and the analogous ones for the hypersurface $V^{*_{n}}$ it is seen at once that $M_{n} g^{1 / 2}=M_{n}^{*} g^{* 1 / 2}$. Hence subtracting equation (4.4) from equation (4.5) yields

$$
\int_{V^{n}} \sum_{\alpha, \beta=1 ; \alpha \neq \beta}^{n} \frac{f^{1 / 2}}{f_{\alpha \alpha} f_{\beta \beta}}\left[\left(b_{\beta \beta} b_{\alpha \alpha}^{*}-b_{\alpha \beta} b_{\beta \alpha}^{*}\right)-\left(b_{\alpha \alpha}^{*} b_{\beta \beta}^{*}-b_{\alpha \beta}^{*} b_{\beta \alpha}^{*}\right)\right] d x^{1} \cdots d x^{n}=0 .
$$

Adding together equation (4.6) and the analogous one by interchanging the two hypersurfaces $V^{n}, V^{* n}$, we obtain

(4.7) $\int_{V^{n}} \sum_{\alpha, \beta=1 ; \alpha \neq \beta}^{n} \frac{f^{1 / 2}}{f_{\alpha \alpha} f_{\beta \beta}}\left[\left(b_{\alpha \alpha}-b_{\alpha \alpha}^{*}\right)\left(b_{\beta \beta}-b_{\beta \beta}^{*}\right)-\left(b_{\alpha \beta}-b_{\alpha \beta}^{*}\right)^{2}\right] d x^{1} \cdots d x^{n}=0$. 
From the assumption $\sum_{\alpha=1}^{n} r_{\alpha}=\sum_{\alpha=1}^{n} r_{\alpha}^{*}$, equation (2.19) and the analogous one for the hypersurface $V^{* n}$, we have

$$
\sum_{\alpha=1}^{n} \frac{1}{f_{\alpha \alpha}}\left(b_{\alpha \alpha}-b_{\alpha \alpha}^{*}\right)=0,
$$

and therefore

$$
\begin{aligned}
\sum_{\alpha, \beta=1 ; \alpha \neq \beta}^{n} \frac{1}{f_{\alpha \alpha} f_{\beta \beta}}\left(b_{\alpha \alpha}\right. & \left.-b_{\alpha \alpha}^{*}\right)\left(b_{\beta \beta}-b_{\beta \beta}^{*}\right) \\
& =\sum_{\alpha, \beta=1}^{n} \frac{1}{f_{\alpha \alpha} f_{\beta \beta}}\left(b_{\alpha \alpha}-b_{\alpha \alpha}^{*}\right)\left(b_{\beta \beta}-b_{\beta \beta}^{*}\right)-\sum_{\alpha=1}^{n} \frac{1}{f_{\alpha \alpha}^{2}}\left(b_{\alpha \alpha}-b_{\alpha \alpha}^{*}\right)^{2} \\
& =-\sum_{\alpha=1}^{n} \frac{1}{f_{\alpha \alpha}^{2}}\left(b_{\alpha \alpha}-b_{\alpha \alpha}^{*}\right)^{2} .
\end{aligned}
$$

Thus equation (4.7) is reduced to

$$
\int_{V^{n}}\left[\sum_{\alpha=1}^{n} \frac{1}{f_{\alpha \alpha}^{2}}\left(b_{\alpha \alpha}-b_{\alpha \alpha}^{*}\right)^{2}\right.
$$

$$
\left.+\sum_{\alpha, \beta=1 ; \alpha \neq \beta}^{n} \frac{1}{f_{\alpha \alpha} f_{\beta \beta}}\left(b_{\alpha \beta}-b_{\alpha \beta}^{*}\right)^{2}\right] f^{1 / 2} d x^{1} \cdots d x^{n}=0 .
$$

It is obvious that the integrand of equation (4.9) is non-negative, and therefore equation (4.9) holds when and only when

$$
b_{\alpha \beta}=b_{\alpha \beta}^{*}
$$$$
(\alpha, \beta=1, \cdots, n),
$$

from which, equations (2.11), (2.17), and the analogous ones for the hypersurface $V^{* n}$ we obtain that $g_{\alpha \beta}=g_{\alpha \beta}^{*}(\alpha, \beta=1, \cdots, n)$. Hence the proof of the theorem is complete.

\section{REFERENCES}

1. S. S. Chern, Topics in differential geometry (Mimeographed notes), Princeton, Institute for Advanced Study, 1951.

2. L. P. Eisenhart, Riemannian geometry, Princeton, 1926.

3. W. V. D. Hodge, The theory and applications of harmonic integrals, 2d ed., Cambridge, 1952.

4. C. C. Hsiung, Some integral formulas for closed hypersurfaces, Math. Scand. vol. 2 (1954) pp. 286-294. 41-46.

5. - A theorem on surfaces with a closed boundary, Math. Zeit. vol. 64 (1956) pp.

6. T. Kubota, Über die Eibereiche im n-dimensionalen Raume, Science Reports of the Tôhoku Imperial University (1) vol. 14 (1925) pp. 399-402.

LEHIGH UNIVERSITY, Bethlehem, Pa. 